\title{
Design and Implementation of Traffic Lights Control System Based on FPGA
}

\author{
Qing Fangzhou \\ School of physics and electronic engineering \\ Qu Jing Normal University \\ Kunming, China \\ e-mail: lihongye1024@163.com
}

\author{
Jun Yang \\ School of Information Science and Engineering \\ Yunnan University \\ Kunming, China \\ e-mail: junyan@ynu.edu.cn
}

\begin{abstract}
Traffic lights are common in daily life's digital systems, the design of traffic lights need to use many basic functional units of digital systems. Traditional digital circuit design is based on the design of integrated circuit in small or medium scale, which has the limitations of needing too many circuits, complex wire, high failure rate, not easy to debug and the success rate is low and so on. This paper implements a traffic light control system based on FPGA under the environment of Quartus II software to overcome the limitations of traditional digital circuit design, which include needing too many circuits, complex wire, not easy to debug, and high failure rate and so on. This paper achieves the control system of traffic lights by using VHDL hardware description language. We verified the correctness and validity of the traffic lights by compiling and simulating the programming downloaded from the programmable logic devices. This traffic lights has the characteristics of high speed, low power consumption and low cost which can be widely used in the market.
\end{abstract}

Keywords- FPGA; Quartus II; EDA technology; Digital Systems;

\section{INTRODUCTION}

Traffic lights are common in daily life's digital systems, the design of traffic lights control is also a classic problem in the digital circuit design[1].

Traditional digital circuit design is based on the design of integrated circuit in small or medium scale, which has the limitations of needing too many circuits, complex wire, high failure rate, not easy to debug and the success rate is low and so on[2,3].

With the development of EDA technology, we can solve the existing problems of the traditional design to a large extent with FPGA as the carrier and the large-scale programmable logic devices as the design tool of developing software.

FPGA (Field Programmable Gata Array), which is a kind of semi-custom circuits of ASIC (Application Specific Integrated Circuits) field [4].

The development of FPGA products are simple, convenient, and has the features of high speed, high precision, reconfigurable, short development cycle and low cost, so the design of traffic lights system based on FPGA, and applying them to related products applications, have certain practical significance and application value.
VHDL hardware description language has a standardized programming structure, multi-level simulation test means, powerful system description capabilities, and flexible statement expressing style[5].

These features above greatly simplify the tasks of hardware design, and improve design's efficiency and reliability, so it has become the primary option of hardware description language in the electronic system design field[6].

In this paper, we design the traffic lights control system based on FPGA, achieve a traffic light controller of crossroad formed by the intersection of a main road and a branch road, and the traffic light control system by using VHDL hardware description language.

\section{DESIGN OF TRAFFIC LightS CONTROL SySTEM BASED ON FPGA}

\section{A. State transition diagram of traffic lights control modules}

The state transition diagram of traffic lights control module is shown in Fig .1.

Where green light of main road, red light of branch road A; Yellow light of main road, red light of branch road B; Red light of main road, green light of branch road C; Red light of main road, yellow light of branch road D; Red light of main road, red light of branch road E. MY, MR, MG represent the main road is yellow light, red light and green light respectively. SM represents there are vehicles on the main road. SB represents there are vehicles on the branch road. EN_URG represents emergency signal. The condition of conversing A, B, C, D to E is EN_URG = 0; While the condition of conversing $\mathrm{E}$ to $\mathrm{A}, \overline{\mathrm{B}}, \mathrm{C}, \mathrm{D}$ is EN_URG $=1$. 


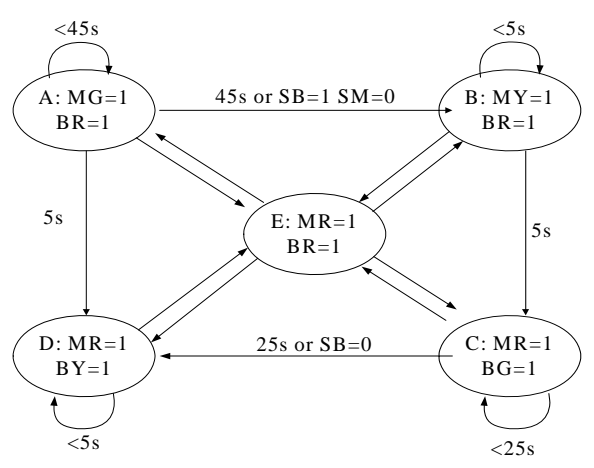

Figure 1. The state transition diagram

\section{B. Design of the function modules and the traffic lights control system}

By analyzing the general design requirements of traffic lights and the transition diagram of basic functions mentioned above, the system designed in this paper consists of five unit circuit components: traffic lights' control unit, display control unit, timing units (including 3 portions: CNT45s, CNT25s, CNT5s), display unit, and decoding unit[7,8].

The overall block diagram of system design is shown in Fig .2.

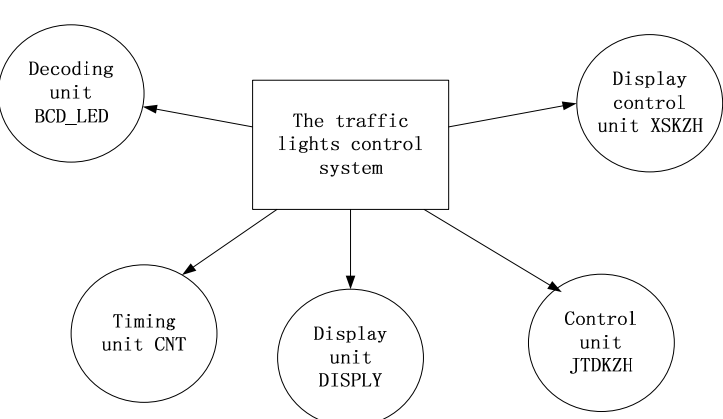

Figure 2. Overall block diagram of system design

The Control unit JTDKZH: This unit mainly sends control signals to the main road and branch road, and sends enable control signals EN45, EN25, EN05m, EN05b to the timing units CNT45s, CNT25s, CNT5s and the display control unit XSKZH simultaneously according to the input signals SM, SB, at the main road and branch road, the clock signal CLK, and the emergency signal EN-URG. The module diagram of JTDKZH is shown in Fig .3.

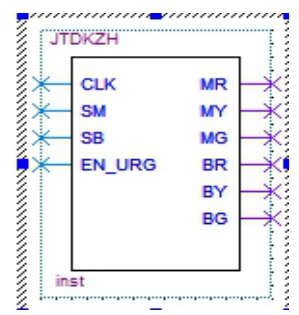

Figure 3. JTDKZH module

The timing units CNT45s, CNT25s, CNT5s achieve the timing of 45 seconds, 25 seconds, and 5 seconds respectively. The timing is achieved by the requirements of enable control signals EN45, EN25, EN05m, EN05b and the emergency signal EN-URG sent by SM, SB, CLK and JTDKZH unit, and then transfer it to the display control unit XSKZH. The module diagram of CNT45s, CNT25s, CNT5s are shown in Fig .4, Fig .5, and Fig .6 respectively.
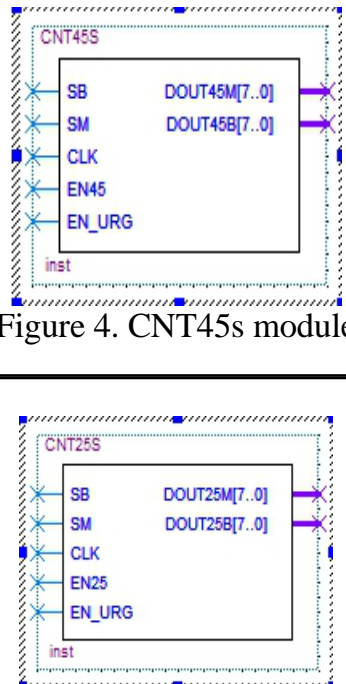

Figure 5. CNT25s module

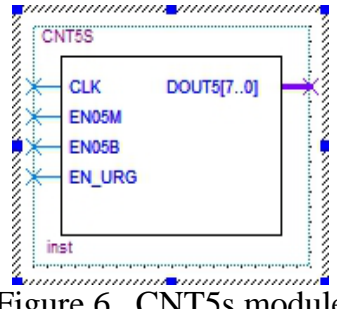

Figure 6. CNT5s module

The display control unit XSKZH: According to the enable control signals EN45, EN25, EN05m, EN05b, and the emergency signal EN-URG sent by the control unit JTDKZH, the system selects the output from the timing units CNT45s, CNT25s, CNT5s and transmit it to the display unit DISPLY. The module diagram of XSKZH is shown in Fig .7.

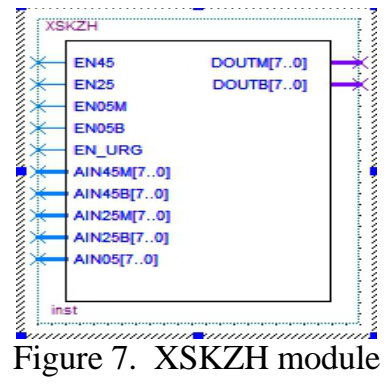

The display unit DISPLY: Transferring the data sent by the display control unit XSKZH to corresponding segment selection and bit selection signals of the seven segment digital tube. The segment selection signal dynamically scan corresponding digital tube, and the bit selection signal will be output to BCD_LED. The module diagram of DISPLAY is shown in Fig .8. 


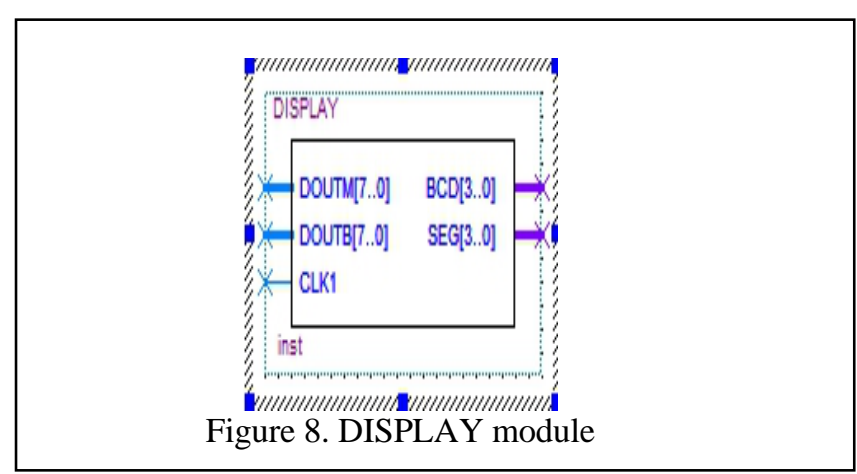

The decoding unit BCD_LED: This unit mainly conducts seven segments decoding to the bit selection signals sent by the display unit DISPLY, in order to display the correct results on the digital tube. The module diagram of BCD_LED is shown in Fig .9.

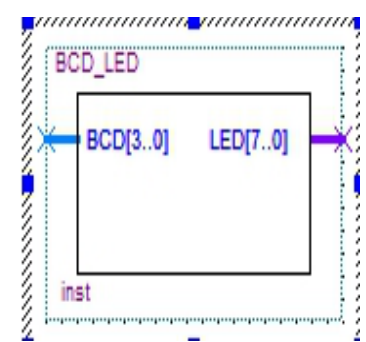

Figure 9. BCD̄_LED module

Through simulating seven units respectively, establishing the symbol file, and creating a new project, we can get the top-level file figure of this system, which is shown in Fig. 10.

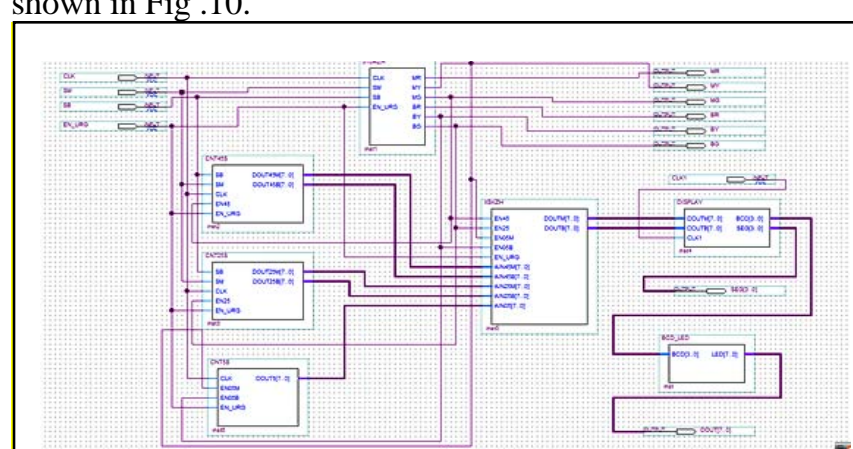

Figure 10. Top-level file figure of the traffic lights system designed

III. SimUlATION OF TRAFFIC LightS CONTROL SYSTEM AND ANALYSIS OF SIMULATION RESULTS

Fig .11 is the system simulation waveform when $S M=1$, and $\mathrm{SB}=1$. It can be seen that the branch road is red light and main road is light green at the beginning; Suddenly the EN_URG $=0$, the red lights of main road and branch road lit up; When EN_URG = 1, the main road turns green light, the branch road turns red light; After 45 seconds, the branch road is still red light, while the main road turns yellow light for 5 seconds, and then the branch road turns green light, and the main road turns red light. Through the analysis, we know that the system satisfy the design requirements.

In addition, each state will be counted once more since the program statement IF CLR='0' THEN $S:=0$ in the JTDKZH module. So the green light will last 46 seconds and the red light will lasts 26 seconds of the main road when the yellow light last 6 seconds. The extra second just be displayed as zero, and which is focused on the point of yellow turning red. In this way, people will not rush through the road when yellow turns red, which can prevent traffic accidents[9].

While Fig .12 and Fig .13 are the partial enlarged system simulation waveform, the two interceptions show the data display before and after of EN_URG becomes zero. We can see that the data displayed is correct, and the display data does not change during the change, while after the change, the display numbers are 25 and 20, and become 24 and 19 when the conversion of red and green happened at 390 seconds, which meet our design requirements.



Figure 11. System simulation waveform of the traffic lights

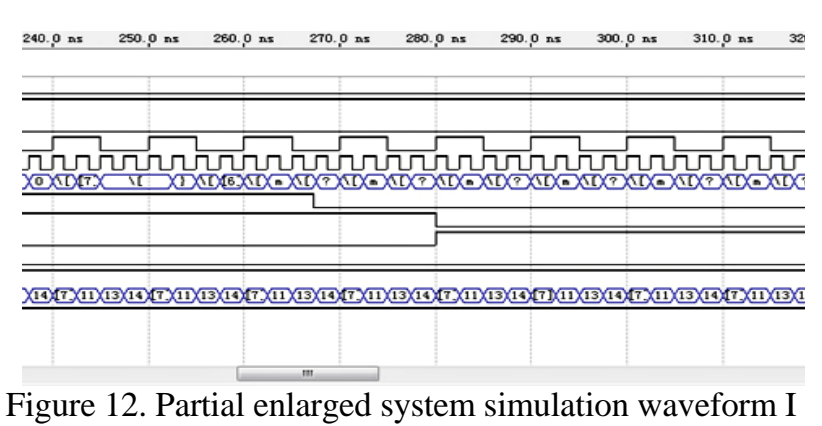

Figure 12. Partial enlarged system simulation waveform I

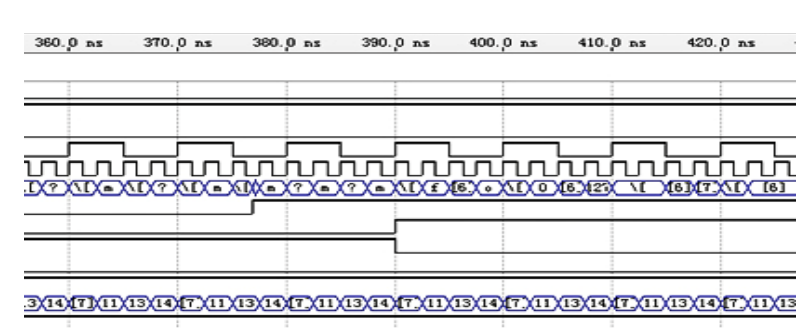

Figure 13. Partial enlarged system simulation waveform II

\section{SUMMARY}

In this paper, we design and implement a traffic lights control system, which can be used to control the signal lights of a north-south and east-west direction intersecting crossroads[10]. 
The traffic lights control system is divided into seven parts after analyzing general design requirements, and achieve the design of the control module with the state transition diagram and the control system with VHDL language.

We verified the correctness and validity of the traffic lights control system by compiling and simulating the programming. We consider that putting this design into production and into the market in future.

\section{ACKNOWLEDGMENT}

This work was financially supported by the Yunnan Re form Project Foundation "Research and Exploration in co mputer architecture Experimental Teaching Content syste m" (X3113008).

\section{REFERENCES}

[1] Chen X . FPGA application experiments.National Defense Industry Publications, China.2013.

[2] Cui W.Design and Analysis of High Performance FPGA System Timing,Microcomputer Applications, 2. 2014.
[3] Liu Y.C. \&T.Y. Wang.Design of traffic light control system based on FPGA.Journal of Henan Institute of Science and Technology(Natural Sciences $\quad$ Edition).41(4):6773.DOI:10.3969/j.issn.1008-7516.2013.

[4] R.Gao.Design of traffic light circuit based on FPGA. PUBLIC COMMUNICATION OF SCIENCE \& TECHNOLOGY.(3):8484,81. 2014.

[5] Song W\&Y.L. Song .Design of traffic light control system based on FPGA.Electronics World.(20):144-144.2013.

[6] Wu J.Altera FPGA/CPLD design (Advanced), Tsinghua University Publications, China.2011

[7] Yuan H.L et al.Design and implementation of traffic light based on FPGA.Electronics Quality.(3):15-19.DOI:10.3969/j.issn.1003-0107. 2013

[8] Yang J. SOPC Practice Guide Based on FPGA.Science Publications, China. 2010

[9] Xu Cheng, Tan Naiqiang, Liu Yan et al. computer applications, Lab (5): 2010,30

[10] Yin Jialin, Li Jing, Wang Jing et al. Based on a timer PLC traffic light control system research. technology, 2013, (8): 6365.DOI:10.3969/j.issn.1001-7119.2013.08.023. 